\section{SOCIOLOGÍA DEL PARTO. Conflictos asistenciales en el marco del "paradigma tecnocrático"}

\author{
José Manuel Hernández GarRe \\ Universidad Católica de Murcia. España \\ jmhernandez@ucam.edu
}

\author{
Paloma Echevarría Pérez \\ Universidad Católica de Murcia. España \\ pechevarria@ucam.edu
}

Cómo citar este artículo / Citation: Hernández Garre, J. M. y P. Echevarría Pérez. 2016. "Sociología del parto. Conflictos asistenciales en el marco del 'paradigma tecnocrático'”, Revista Internacional de Sociología, 74 (1): e025. Doi: http://dx.doi.org/10.3989/ris.2016.74.1.025

\section{SOCIOLOGY OF CHILDBIRTH. Care conflicts under the "technocratic paradigm"}

Copyright: ( 2016 CSIC. Este artículo de acceso abierto distribuido bajo los términos de la licencia Creative Commons Attribution-Non Commercial (bycn) Spain 3.0.

Recibido: 14/10/2014. Aceptado: 03/03/2015. Publicación online: 05/02/16

\section{Abstract}

\section{Resumen}

El objetivo del artículo ha sido explorar, desde la óptica de las propias parturientas, los posibles conflictos surgidos entre las necesidades maternas y las rutinas médicas propias del parto hospitalario e intervencionista. La metodología elegida ha sido la realización de una serie de entrevistas semiestructuradas, cuyos resultados muestran fundamentalmente tres tipos de tensiones asistenciales: la interacción asimétrica, la subordinación tecnológica y la asunción de praxis de "abuso institucional". La conclusión final ha sido que estamos ante un ritual medicalizado impregnado de vivencias de obediencia pasiva, en el que el miedo al peligro siembra la desconfianza corporal, a la vez que la voluntad de la mujer es moldeada por los expertos. Realidad que las parturientas asumen como inevitable, e incluso demandan, como consecuencia de la seguridad que les aporta el control biotecnológico, una vez asumidos los discursos clínicos que representan al parto como un proceso patológico y de riesgo.

\section{Palabras Clave}

Experiencias maternales; Parto hospitalario; Parto intervencionista; Parto medicalizado.
The aim of this article is to explore, from the point of view of the women who have just given birth, possible conflicts between maternal needs and medical routines typical from interventionist hospital births. The chosen methodology has been a set of semi-structured interviews, and the result shows principally three types of relief aid: the asymmetrical interaction, technological subordination and the acceptance of "institutional-abused" praxis. The final conclusion is that we have a medicalized ritual influenced by passive obedience experiences, where the fear creates distrust, while the women wishes are moulded by the expert's. This fact is assumed by the women who have just given birth as inevitable, and they even demand it, as a result of the safety given by the biotechnological control, and once they have assumed the clinical discourses which present the childbirth as a pathological and risk process.

\section{KEYWORDS}

Maternal Experiences; Hospital Birth; Interventionist Birth; Medicalized Birth. 


\section{INTRODUCCIÓN}

Uno de los fenómenos biológicos que ha experimentado una mayor transformación en las últimas décadas ha sido el del parto. Hasta el punto de que tras la segunda guerra mundial fue trasladado a los hospitales, siendo diana del escrutinio sistemático de toda una serie de controles cuyo objetivo fue representarlos biométricamente. Desde ese momento, la reproducción se fue convirtiendo en un fenómeno altamente tecnológico (Oakley 1984) que abarcaba desde las técnicas de fecundación asistida o los controles ecográficos, hasta las amniocentesis o los métodos de inducción de parto. En este contexto los partos se han convertido en trances tecnológicos, en los que, a veces, se tiende a una excesiva medicalización e intervencionismo bajo discursos como el de la evitación del riesgo o la anulación del dolor (Montes 2007). Se trata de una nueva cosmovisión del nacimiento que ha sido bautizada por autores como Davis-Floyd (2001) con el nombre de "paradigma tecnocrático", y caracterizada por autoras como Nancy Stoller (1974) con atributos como la medicalización, la institucionalización, la fragmentación del cuidado, la tecnologización o el monopolio médico. Estamos, en definitiva, ante una "cultura del nacimiento" (Montes 2007) o "etnoobstetricia"1 (Mcclain 1975) hospitalaria e intervencionista (Blázquez 2009) que en países con acceso a tecnología sanitaria se ha ido instituyendo en la modalidad de parto más común, mayoritaria y legitimada (Hernández 2011; Blázquez 2009; Montes 2007; Martin 1987). Fenómeno de estudio documentado en los últimos años a través de toda una serie de exploraciones etnográficas (Hernández 2011; Blázquez 2009; Imaz 2008; Montes 2007; Bodoque 1996; Esteban 1992; Jordan 1993; Joaquin 1983; Knibiehler y Fouquet 1983; Pizzini 1981) que, desde una perspectiva crítica, lo han descrito como un proceso rutinario, uniforme, sistemático, ritualizado, burocrático y autoritario.

Las evidencias científicas hablan, pues, de una tendencia a la medicalización de los partos que no ha pasado desapercibida a entidades sanitarias como la Organización Mundial de la Salud (OMS). Esta ha defendido en diferentes documentos (Organización Mundial de la Salud 1985; 1996) el derecho de las madres a un cuidado humanizado y a un papel central en todos los aspectos de su atención, incluyendo la participación activa en la planificación, ejecución y evaluación de su asistencia. Ha hecho, además, hincapié en la promoción de investigaciones que evalúen las praxis y tecnologías de atención al parto más adecuadas, apostado por la promoción de un itinerario asistencial menos medicalizado que evite

1 Los términos "cultura del nacimiento" o "etnoobstetricia" se refieren a las diferentes praxis e itinerarios asistenciales que cada cultura particular establece para dar cobertura a los nacimientos. la tasa de cesáreas e inducciones de parto injustificadas, y promocione la consecución de partos normales (OMS 1985). El objetivo fundamental ha sido la consecución de una madre y un niño sanos, con el menor nivel de intervención posible y de la manera más segura, promocionando praxis clínicas como la participación activa de las madres, el respeto a la intimidad, la elección del lugar del parto, el derecho al acompañamiento, la movilidad, el apoyo emocional efectivo o la toma de decisiones intraparto. Se trata, en definitiva, de un afrontamiento igualitario entre las prácticas biomédicas y otras consideradas como alternativas. De un nuevo análisis crítico de las rutinas clínicas sistemáticas y de los modelos hegemónicos de atención, desde el que se respeta la complementariedad entre los saberes médicos y los recursos naturales maternos (OMS 1996). Esta nueva perspectiva de la OMS se fundamenta, además, en toda una serie de estudios y evidencias científicas (Olsen 1997; Campbell y Macfarlane 1987; Tew 1985) que muestran cómo el excesivo intervencionismo en los partos normales puede, incluso, incrementar la morbilidad de las madres y los bebés. La conclusión de estas investigaciones es que hay que racionalizar el uso indiscriminado de la tecnología en el parto, ya que la intervención sistemática y protocolizada en los nacimientos de bajo riesgo aumenta las complicaciones materno-fetales, mientras que las intervenciones puntuales en determinadas situaciones patológicas intraparto pueden, sin embargo, salvar la vida de las madres y sus bebés.

En este contexto han surgido en España una serie de documentos emitidos por el Ministerio de Sanidad y Política Social (2010a; 2010b) que insisten en la necesidad de evitar la medicalización excesiva de los partos de bajo riesgo, racionalizando las tasas de cesáreas y partos instrumentados y respetando los tiempos de duración del parto normal. Documentos igualmente secundados por sociedades científicas, como la Federación de Asociaciones de Matronas de España (FAME), que en su última guía de práctica clínica (2008) también ha apostado por disminuir la intervención en el nacimiento, situando la excelencia de éste en aspectos como la actitud expectante, la promoción de un entorno humanizado y el respeto a las necesidades emocionales de las madres.

Estamos pues ante un auténtico debate social entre lo que podríamos catalogar como cosmovisión intervencionista y humanista del parto ${ }^{2}$. De un objeto de estudio en el que, tal como se ha dicho, la propia OMS ha hecho especial hincapié, en los últimos

2 De una parte están las perspectivas clínicas, basadas en literatura biomédica, que apuestan por la biointervención y la disminución de riesgos (Sociedad Española de Obstetricia y Ginecología 2008a). De otro lado está la literatura divergente -Michael Odent (1990; 2011); Laura Gutman (2006)- o las asociaciones de madres -"El parto es nuestro"- que cuestionan las versiones biomédicas oficiales defendiendo un abordaje más humanizado. 
años, con el objetivo de esclarecer la forma en que se está aplicando la tecnología y el control profesional en estos rituales medicalizados del nacimiento. Contexto investigador en el que se enmarca el presente estudio, cuyo objetivo ha sido explorar, desde la propia óptica de las madres, los posibles conflictos surgidos entre sus necesidades y las rutinas medicalizadas propias de este patrón cultural de parto. El análisis se ha hecho desde un posicionamiento teórico postmoderno-reflexivo ${ }^{3}$ que, desde la perspectiva de género ${ }^{4}$, debate sobre los discursos hegemónicos biomédicos característicos de la modernidad y su implicación en las representaciones y vivencias maternales. Ello ha permitido profundizar en dimensiones del fenómeno diferentes a las que clásicamente abordan las investigaciones clínicas -los marcadores epidemiológicos-, ha permitido, en definitiva, examinar la posible tensión entre las rutinas asistenciales y las propias experiencias maternas. En este sentido, pensamos que las mayores aportaciones del artículo están en la categorización que se hace de los conflictos asistenciales surgidos en el marco de este paradigma asistencial y en el hecho de que estos se codifiquen desde la propia óptica de las madres. Nos parece, pues, pertinente el análisis que aquí se plantea, máxime cuando la humanización del parto dista todavía de ser una realidad consolidada en los hospitales, a pesar de las recomendaciones y buenas intenciones de las diferentes instituciones.

\section{Metodología}

La naturaleza de la investigación determinó la elección de un enfoque cualitativo que recogiera los aspectos vivenciales de los partos hospitalarios. Por ello, el estudio se decantó por abordar el fenómeno desde la perspectiva de las madres, obteniendo información decisiva sobre cómo interpretaban, percibían y experimentaban los acontecimientos en torno al nacimiento. Fue posible, así, el acceso a la información desde la perspectiva "emic", partiendo de la base de que las madres tenían su propio punto de vista, lo que implicaba que la realidad debía ser descrita y analizada a partir de lo que decían sobre sí mismas y del contexto que les rodeaba (Menéndez 2000). Desde este enfoque se tuvo "la oportunidad

3 Posicionamiento representado por autores como Gilles Deleuze, Jean-François Lyotard, Michel Foucault o Jacques Derrida desde el que se cuestionan las verdades absolutas defendidas desde las ideologías modernistas, y se piensan que la certeza no es más que una verdad relativamente interpretada; perspectiva desde la que se cuestiona el quehacer de las diferentes instituciones sociales y políticas.

4 Movimiento ideológico iniciado por autores como Margaret Mead, Simone Beavouir o John Money, que reflexiona sobre todas las representaciones culturales y sociales que construyen la identidad masculina y femenina. Se trata de examinar el impacto del sexo en las oportunidades de las personas, sus roles sociales y las interacciones mutuas. de caracterizar con mayor precisión las representaciones, experiencias y prácticas sociales" (Osorio 2001: 43) percibidas por las madres, "obteniendo datos más estratégicos y proponiendo interpretaciones más correctas" (Menéndez y Di Pardo 1996: 60-61). La técnica de recogida de datos fue la de las entrevistas, centrando el foco de atención, como se ha comentado, en el análisis de los posibles conflictos surgidos entre las necesidades de las madres y las praxis típicas de este patrón asistencial intervencionista del parto, característico del "modelo biomédico hegemónico". El contexto del estudio fue el paritorio de un hospital público del sur de España que da cobertura a una extensa área de Salud. El centro fue elegido por representar un ejemplo paradigmático de medicalización de los partos, realidad reflejada tanto por su itinerario asistencial, caracterizado por el férreo control y medicalización de los mismos, como por sus altos estándares de cesáreas (31\%), inducciones de parto $(25 \%)$ y episiotomías $(50 \%)^{6}$. Marcadores clínicos característicos de las "culturas del nacimiento" medicalizadas que se encuentran muy por encima de los estándares recomendados por la OMS, que recomienda un máximo admisible de un $15 \%$ de cesáreas y un $10 \%$ de inducciones y episiotomías (OMS 1985). Por razones de confidencialidad, y solicitud expresa del centro, se ha optado por mantener el anonimato del mismo.

Las entrevistas fueron semiestructuradas, intentando en todo momento la producción de una conversación continúa con una línea argumental no excesivamente fragmentada (Grele 1991; Taylor y Bogdan 1987). Se realizaron en los primeros cinco días posparto, asegurándose así un relato nítido y reciente, en el periodo comprendido entre el 1 de febrero y el 31 de marzo del año 2010, tras la autorización del centro y dado el consentimiento informado de las madres. La confidencialidad de las mujeres se garantizó en todo momento, realizándose los encuentros en un espacio privado -la sala de lactancia de la planta de maternidad- a la vez que se les asignaba un código encriptado que garantizaba su anonimato. Para determinar el tamaño de la muestra se siguió el criterio de saturación, entendido este como la situación a la que se llega después de haber recogido el relato de un determinado número de informantes (D. Bertaux e I. Bertaux 1993). La información se saturó al llegar al número de 20 participantes, con un total de $280 \mathrm{mi}-$ nutos, que se editaron con el programa de gestión de archivos sonoros Digital Voice Editor 3.1., siendo íntegramente trascritos con el software de reconocimiento de voz Dragon Naturally Speaking Preferred 10.

Para dar validez interna a la muestra se tuvo en cuenta, además de la consecución de un elevado

5 Término acuñado por el antropólogo Eduardo Menéndez para referirse a la hegemonía que ejerce el sistema biomédico occidental sobre otros sistemas médicos considerados como subalternos.

6 Las estadísticas reflejan la media aritmética para cada uno de los parámetros en el periodo comprendido entre 2008 y 2013. 
nivel de saturación del discurso, el principio de intersubjetividad. En este sentido, la heterogeneidad de las gestantes del área de estudio, y la multiplicidad de factores vivenciales y biográficos ajenos a la naturaleza de la investigación, determinó la necesidad de homogenizar la misma, siguiendo para ello los siguientes criterios de selección: edad entre 15 y 35 años (74 \% de la población), nacionalidad española (67\% de la población), situación de pareja fija y heterosexual, nivel socioeconómico estable, primiparidad (54\% de la población), embarazo sin patología (76 \% de la población) y gestación biológica deseada $(91 \% \text { de la población })^{7}$. El objetivo fue evitar sesgos que pudieran distorsionar las experiencias ligadas a la dinámica medicalizadora, propia de este paradigma asistencial tecnocrático, reflejando así experiencias derivadas de otras circunstancias personales y socioculturales que no se pretendían abordar en este estudio.

En este sentido, para una mayor clarificación de los criterios de selección, hay que hacer notar que la mayoría de los protocolos catalogan los embarazos en edades reproductivas inferiores a 15 años o superiores a 35 como de riesgo (Sociedad Española de Obstetricia y Ginecología 2008b), considerando a las primeras demasiado jóvenes para afrontar la maternidad, y las segundas, gestantes añosas susceptibles de embarazos potencialmente complicados. Al acotar las edades se ha pretendido, pues, minimizar la influencia de perspectivas ajenas al estudio, relacionadas con la consideración especial de riesgo, la movilización de recursos personales, la protocolización de un itinerario clínico específico, que conlleva un mayor control y visitas, o la mayor interiorización materna de los discursos biomédicos de riesgo. En cuanto al acotamiento en base a nacionalidad, partió del hecho de que las experiencias del parto están claramente influidas por los factores culturales de origen, condicionando, en cierta medida, sus discursos y representaciones (Luque y Oliver 2005). En este sentido, ser extranjera influye en factores como el nicho laboral, la cosmovisión del parto, los hábitos alimenticios, las perspectivas de prevención y control de la salud, la expresión del dolor, las creencias religiosas o las barreras idiomáticas, factores ajenos a la investigación y que sin duda condicionan las experiencias del parto. En otro orden de cosas, hay que apuntar que las mujeres sin pareja fija pueden presentar situaciones emocionales añadidas, mientras que las parejas del mismo sexo presentan sus propios conflictos, discursos, prácticas e itinerarios clínicos (Schwarz 2008), circunstancias que también pueden distorsionar la vivencia del parto al margen

7 Las cifras hacen referencia a los porcentajes recogidos en los registros del centro, tomando como población el total de mujeres que dieron a luz en el año 2010. No existen registros sobre el tipo de pareja y el nivel socioeconómico por lo que el acceso a la información se consiguió mediante un sondeo previo a los participantes del estudio. de los objetivos exploratorios del estudio. Por otro lado, somos conscientes de que la determinación de la situación socioeconómica plantea ciertas dificultades, ya que en esta influyen variables tan variopintas como los ingresos económicos, el nivel de estudios o la ocupación (Borrel 2004). En este caso la decisión de incluir únicamente a las madres con situación económica estable, con independencia de su nivel de estudios o situación laboral, vino motivada por la necesidad de minimizar preocupaciones adicionales relacionadas con la escasez de recursos, que pudieran influir negativamente en la aceptación y vivencia del embarazo y parto. A ello hay que añadir que la mayoría de los protocolos clínicos incluyen el nivel socioeconómico bajo como uno de los factores sociodemográficos de riesgo (Sociedad Española de Obstetricia y Ginecología 2008b), susceptible de provocar complicaciones en el embarazo, realidad que influye en los itinerarios y en las representaciones profesionales y maternas. También hay que señalar que las mujeres multíparas presentan ciertas preconcepciones relacionadas con sus experiencias anteriores en los servicios sanitarios (Baroni, Vilela, Scorsolini 2013), configuraciones de partida ajenas al estudio y que pueden también condicionar de forma positiva o negativa las ulteriores vivencias. Por último, se quiere matizar que la decisión de incluir exclusivamente a madres sin patología durante el embarazo, y con embarazo deseado, estuvo motivada por la conveniencia de evitar elementos ajenos a la investigación, como conflictos emocionales ligados a las patologías o a la negación de la gestación, que sin duda incrementan la percepción de riesgo y disminuyen la aceptación del proceso. Además, hay que tener en cuenta que las sociedades científicas catalogan también a estas gestantes con la etiqueta de riesgo (Sociedad Española de Obstetricia y Ginecología 2008), lo que conlleva itinerarios clínicos específicos que influyen decisivamente en sus percepciones del parto.

En cuanto al análisis del discurso, este se realizó atendiendo a las siguientes categorías: tipo de interacción con los profesionales, información y apoyo emocional recibido, uso de tecnología durante el proceso y respeto a la dignidad de las madres. Los discursos maternos fueron bastante homogéneos para cada una de las categorías de análisis, y aunque con ciertas discrepancias, dibujaron unos conflictos y tensiones maternas bien definidos. Para su categorización se utilizó el programa MAX.QDA (versión 2) de gestión de datos cualitativos, seleccionando los testimonios en base a las categorías y objetivo del estudio, por lo que se dio prelación a los relatos que reflejaban las tensiones fundamentales entre las necesidades maternas e institucionales. Durante el análisis se tuvieron en cuenta las propuestas de Jesús Ibáñez (1979), sobre la interpretación del discurso, que recomiendan introducir un orden en el mismo: lectura intensiva, toma de notas, 
análisis e interpretación, listados, primer epígrafe del informe, codificación, agrupación y categorías temáticas. También se siguieron las recomendaciones de Jociles Rubio (2005), respecto a la eficacia simbólica de los alegatos en los contextos en que se producen, redactando el artículo en torno a la prolífica literatura existente.

El estudio presenta ciertas limitaciones, y en este sentido los resultados no pretenden ser categóricos ni extrapolables indefectiblemente a todas las madres, sino al perfil materno recogido en los criterios de selección, limitándose además la exploración a los posibles conflictos surgidos durante la interacción con las rutinas médicas propias del parto hospitalario e intervencionista. Por ello, el análisis no se ha centrado en otros focos de interés como los efectos positivos de esta "cultura del nacimiento", las perspectivas de otros colectivos implicados en el proceso, como los propios profesionales, u otros posibles perfiles maternos. En este sentido, cabe destacar lo apuntado por Oriol Romaní (1992), al afirmar que las relaciones durante la investigación nunca son totalmente neutras ni simétricas, sino que responden indudablemente a los posicionamientos de los implicados en la investigación, entre ellos los del científico. Otra de las limitaciones de la exploración etnográfica es la total extrapolación de los resultados, el problema surge por la dificultad de abordar un fenómeno de estudio a través de lo que Marcel Mauss (1966) denominara como una "individualidad tipo", es decir, un informante representativo de esa cultura. El problema se presenta por la inmaterialidad de la cultura, lo que implica su discernimiento a través de los ojos de un informador que, aunque sea una expresión ideal de esa cultura, también presenta cierto grado de discrepancia con la misma. Por tanto, los resultados solo se pueden intuir por medio de una doble distorsión, la de las informantes y la del propio observador. Pese a las limitaciones, creemos que el estudio ha conseguido recoger, con suficientes garantías, las tensiones más comunes relatadas por el perfil de madres del estudio como consecuencia de su relación con los sistemas hospitalarios intervencionistas.

\section{Resultados/Discusión}

Las interacciones, humanas y técnicas, que codifican las experiencias maternales a lo largo del itinerario asistencial, están cargadas de fuerza simbólica, traduciendo los valores culturales hegemónicos del contexto hospitalario en la que se insertan (Bourdieu 1994), por lo que contribuyen al mantenimiento de la estructura social y clínica inmanente. En este sentido, el análisis de las experiencias de las madres relacionadas con las tensiones surgidas en el transcurso de las ceremonias y praxis medicalizadoras del parto, permiten trazar ciertas mundologías dominantes en los ámbitos hospitalarios. Cosmovisiones asistenciales que desde la perspectiva de la tipología de madres seleccionadas para esta investigación se materializan, fundamentalmente, en tres conflictos asistenciales: la interacción asimétrica, la subordinación tecnológica y la asunción de praxis de "abuso institucional". Desmenucemos, pues, cada una de estas experiencias desde la propia óptica de las madres, haciendo notar que a efectos de una mayor agilidad en la redacción se han utilizado indistintamente los nombres genéricos de "mujer", "madre", "parturienta" o "gestante" para referirse exclusivamente a la tipología de madres acotada en los criterios de selección del estudio.

\section{Interacción asimétrica}

A tenor de los testimonios maternos, parece que la relación con los profesionales ${ }^{8}$ se instituye por medio de un vínculo asimétrico, en el que unos ostentan el "saber" que los inviste en expertos, y otros el lugar del "no saber", que los etiqueta como pacientes. Queda así acotado cierto nexo de capitulación, que es alimentado por el hecho de que los técnicos muestran con frecuencia su pericia a través del uso de una jerga clínica que no entienden las madres: "Yo prefiero que me hablen como una persona normal y corriente de pie de calle que como si fuera una máquina, un robot que le vas diciendo: alto y claro, y que digan las cosas a pie de calle, porque nosotros no somos cualificados de la medicina" (xxס). Desde esta configuración asistencial da la impresión de que las protagonistas desempeñan un rol pasivo, desde el que acatan y rara vez cuestionan la esfera de poder de los especialistas, la traducción biométrica de su dimensión biológica: "Técnicamente, hombre tampoco sabemos técnicamente lo que se necesitaba, pero vamos, creemos a nuestro entender que sí, que la atención fue la correcta" (xxu). Se produce, así, cierta invisibilización del conocimiento materno, consecuencia de la monopolización de la salud que, a veces, se hace desde las instituciones sanitarias. A ello se suman los discursos maternos de miedo e inseguridad sobre el propio funcionamiento corporal, realidad que incrementa, a su vez, la dependencia técnica de los expertos: "Tienes miedo como no sabes qué, nada de qué va a venir, ni de cómo lo vas a hacer, yo ahora que lo he visto esto es preferible estar aquí que tener una tensión, a pesar de todo son profesionales" (xxK). Se produce así, en línea con lo apuntado por Juan (1991) y De Miguel (1979), una representación de los cuerpos gestantes en clave

8 Bajo el término de profesionales, expertos, especialistas o técnicos se recogen las dos categorías de asistentes existentes en nuestro contexto: los médicos-obstetras y las matronas. Los discursos maternos no hacían especial diferenciación entre ambos colectivos, haciéndolos coparticipes de una misma representación asistencial. Razón por la que no se ha hecho distinción entre ambos colectivos a lo largo del discurso. 
patológica como consecuencia de la inseguridad que producen las posibles complicaciones que pudieran sobrevenir durante el proceso.

Como expone Taussing (1995), la especial situación se convierte en una herramienta de poder que moldea las voluntades maternas ante la mística de un proceso que se inviste de imprevisible. Llegados a este punto las madres prefieren derivar el control de sus partos a los profesionales, ya que así se sienten más seguras: "Además que no sabes cómo se te presenta el parto, yo pensaba que iba a tener un parto tal y al final pues, pues no" (xxк). De esta manera, las madres acaban viviendo sus partos a través de los ojos de los profesionales, adoptando, inducidas por la propia dinámica asistencial, el rol pasivo y sumiso del que nos hablan autores como Pizzini (1981). Papel litúrgico que es vivido como lo "natural”, al encontrarse en el espacio clínico e institucional de poder de los profesionales: "Estás en un sitio que tienes que hacer lo que ellos hacen, lo que ellos

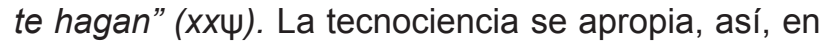
cierta medida, de los propios sentidos maternos, siendo las cosas tal y como ella las define, por lo que el discurso materno ya no será imprescindible desde el mismo momento en que los datos biológicos dictan la realidad objetiva (Menéndez y Di Pardo 1996). Estamos, pues, ante un proceso relacional asimétrico en el que cada vez se "excluye más la palabra de la "paciente"”' (Menéndez 2002: 179), contingencia cuya consecuencia final es la dependencia total del "saber" de los sanitarios (Ortiz 2006; Esteban 1992; Juan 1991): "la atención lo es todo, porque estás en sus manos" ( $x x \lambda)$. Parece que las raíces ideológicas de esta configuración asistencial habría que buscarlas en lo que Foucault (1990) denominara con el término de "biopolítica", es decir, en la pretensión de las instituciones de controlar los procesos reproductivos con el fin de asegurar ciudadanos sanos y productivos. Se trataría, en definitiva, de una estrategia por la que los Estados utilizan los gremios profesionales para controlar, a través de la vigilancia exhaustiva, los procesos corporales en aras de la consecución de una buscada eugenesia social.

Los relatos maternos muestran cómo, desde esta configuración ritual, las decisiones son dictadas por las visiones clínicas, por lo menos mientras se inserten en un itinerario asistencial establecido bajo la aceptación y cumplimiento del quehacer hospitalario: "Decisiones tú no puedes tomar ninguna, las decisiones las toman ellos" ( $x x \Psi)$. Desde la asunción de este rol las rutinas se centran con demasiada frecuencia en las dimensiones biológicas: "Se limitan a entrar, te miran, te exploran y punto, ya está" (xxK). Se trata de un tipo de interacción en la que los profesionales "se dedican a la exploración e interpretación corporal, a valorar los datos de los monitores, analíticas y otras pruebas, mientras otros aspectos "emocionales" ocupan un tiempo reducido" (Blázquez 2009: 238). En este contexto, algunas madres se quejan de un tipo de relación asistencial excesivamente técnica que lleva, a veces, a actuar al margen de sus necesidades emocionales: "Haciendo su trabajo, mirando el monitor y los resultados y tal, pero no les salía ni palabra" (xx入). Como apunta Blázquez (2009), los circuitos clínicos están impregnados de cierta filosofía productivista que hace que el seguimiento, registro y control de los protocolos e itinerarios programados sea lo prioritario, por lo que en ocasiones no se presta suficiente atención a otro tipo de necesidades de las pacientes; lo importante es la biometría, por tanto, los saberes y experiencias de las mujeres ya no son imprescindibles: "Entonces era que tú estás mal, claro, y les haces preguntas y es un monosílabo o un sí, y necesitas más, necesitas más" ( $x x \lambda)$. Se trata de una interacción profesional excesivamente centrada en la biometría del proceso: "Se han dedicado a hacer estrictamente su trabajo, a hacerte la sonrisa porque te la hago, pero aquí tontos no somos" (xxK). Estamos, en definitiva, ante una relación caracterizada en su mayoría por la búsqueda de información fisiológica, la atención en el plano físico (exploraciones, canalizaciones de vías, etc.) y la subyugación de la relación humana a la tecnológica. Realidad que lleva al personal clínico a estar, durante gran parte del itinerario asistencial, más atento a la decodificación de las señales del cuerpo que a la propia paciente (Cunha y Ojeda 2006). Se intuye aquí cierta ideología "posthumanista" que "pretende hacer tanteos [tecnológicos] para averiguar las posibilidades de mejora" (Ferrer 2007: 156) de la propia naturaleza de las gestantes. Cosmovisión desde la que las madres son percibidas como organismos frágiles cuyas prestaciones han de ser maximizadas a través del uso e interpretación de las máquinas.

Parece, pues, en base a los testimonios maternos, que la implantación de esta modalidad de asistencia se ha producido como consecuencia de que las intervenciones profesionales se han centrado casi excesivamente en los úteros maternos, soporte de los fetos, de manera que las madres han ido desaparecido como sujeto emocional: "No al margen de su trabajo no [...] ella venía y me decía que venga, que aligerara, y que empujara, y que ya está, si es que no [...] y venga y es que llevas aquí mucho tiempo, y vamos, $y$ te veías ya un poquito ya forzada, si es que yo no puedo hacer más que lo que hago" ( $x x \Psi)$. Se trata de una tendencia asistencial por la que las pacientes se convierten, a veces, en una herramienta de trabajo en manos de los expertos, de manera que su presencia como sujeto corre el peligro de ser olvidada, ya que los datos hablan por ellas. Como afirma Maroto et al. (2009), estaríamos ante una configuración asistencial rígida y, en ocasiones, poco acomodada a la satisfacción de las necesidades reales de las madres. Paradoja, como apunta Suárez y Puertas (2008), por la que la biología ocupa gran parte de la atención profesional, mientras que las madres manifiestan, sin embargo, 
necesidades y problemas de carácter emocional y afectivo: "Llegas con el miedo, yo era un primer parto, no sabes lo que es, llegas con el dolor y claro que te estén preguntando y arropando y queriendo que estés bien, pues es lo primero que miras, porque llegas con ese miedo, y con el no saber y entonces eso te ayuda bastante a sentirte bien" (xxß). Así, las experiencias de la mayoría de las madres muestran que tiene una mayor relevancia el componente relacional de la asistencia que el técnico (Bravo, Uribe y Contreras 2008): "Más que a la tecnología quizás al parto en sí, que se haga con métodos más avanzados o menos avanzados yo que sé, no [...] pero un poco más sobre todo tema emocional, sí, más que nada porque te encuentras muy sola" (xxס). En este escenario tecnológico las implicadas demandan una mayor presencia de cualidades personales, como cariño y trato agradable, para sentirse protagonistas de su parto (Goberna, Palacios y Banús 2008). Ello implica que el cuidado de los profesionales se lleve a cabo desde una configuración más humanística, caracterizada por un enfoque de la atención menos técnico y más emocional (Merighi, Carvalho y Suletroni 2007; Becerra y Cardoso 2005). Perspectiva humanista y apoyo emocional que, según la evidencia disponible, supone, además, grandes beneficios para la parturienta, aumentando las posibilidades de parto vaginal y disminuyendo el trabajo de parto y la utilización de analgesia (Hodnett et al. 2011; Federación de Asociaciones de Matronas de España 2008; Ministerio de Sanidad y Política Social 2010).

Otro de los aspectos mencionados por las madres del estudio es la forma en que se gestiona la información. En este sentido las madres se sienten, a veces, al margen del proceso que, como ya se ha comentado, se centra excesivamente en el área de poder de la tecnociencia, la fisiología: "Están haciendo pruebas pero nunca te dicen para qué son o qué es lo que te están haciendo, es todo muy mecánico [...] tú estás allí bueno y ahora qué, qué están haciendo" ( $x x \lambda$ ). Las implicadas en el parto, demandan, de esta manera, una mayor información que les reintegre en el conocimiento de la evolución del trance: "Falta información de decirte lo que está pasando, y bueno si hay complicaciones pues que te lo digan" $(x x \lambda)$. El silencio da atribuciones hieráticas al ritual, aumentando el misticismo del que se rodean todas las actuaciones de la ciencia empírica, disparando, a veces, innecesariamente las ansiedades: "Pero si son tres o cuatro horas yo creo que [...] dar una información o algo [...] pero ahí el reloj corriendo y venga, y venga, y venga y claro la cabeza da muchas vueltas [...] creo que falta información" (xxع). En este sentido, como afirma Bodoque (1996), explicar los resultados de las pruebas y las razones de las decisiones sanitarias es la mejor táctica para disminuir el desasosiego que la propia dinámica asistencial crea, convirtiéndose, a su vez, en uno de los requisitos indispensables para que las madres vivan el nacimiento de forma satisfactoria (Bravo, Uribe y Contreras 2008). Pese a ello, la propia mecanización del proceso hace que la información sea en muchas ocasiones escasa (Cunha y Ojeda 2006): "Yo lo veo escaso y a cuentagotas [...] entonces bueno te van dando como golpes, un golpe detrás de otro, y tú te quedas así un poco en shock cada vez que te dan la información" ( $x x \lambda)$.

Pese a todos los conflictos y tensiones asistenciales apuntados hasta ahora, y a las asimetrías en la interacción asistencial -los expertos adoptan roles activos de autoridad y las madres pasivos de acatamiento-, las normas clínicas son asumidas por las madres y vividas como lo "normal". Se produce así, paradójicamente, una buena aceptación de las dinámicas propias del parto hospitalario e intervencionista, reflejando las madres, desde la seguridad que les produce el control biomédico, un grado de conformidad aceptable del mismo (Castro y Muñoz 1999): "Sí estuvo todo bien, la cesárea ha salido bien, la hicieron bien, no he tenido problemas después, y yo con las vías no he tenido problemas ninguno, ni con los asistentes ni nada de eso" (xxع). Aparecen, pues, también, en el relato de las madres, los efectos positivos de este abordaje asistencial; efectos que a la postre minimizan los inconvenientes de la interacción asimétrica característica de este "paradigma tecnocrático".

\section{Subordinación tecnológica}

El simbolismo que subyace en los testimonios de las madres permite interpretar los claro-oscuros que se derivan de la relación tecnológica. Estamos ante un modelo asistencial técnico, en el que los traductores conectan a las madres a un gran arsenal de artefactos mecánicos que aseguran la traducción de las percepciones intuitivas en datos biométricos. No se trata de una simbiosis, sino de cierta invasión de los propios sentidos maternos, en el sentido de que los cables, los bits de las máquinas, sustituyen, y en cierta medida suplantan, las identidades corporales: "La verdad es que estaba enchufada por todos sitios" (xxK). Existe, pues, desde este paradigma clínico, cierta tendencia a abusar de una interpretación biométrica que reduce las experiencias maternas a simple información (Amato 2007), siendo, a veces, las mujeres condicionadas a vivir y sentir aquello que dicta la propia tecnología. Como afirma Duden (1996), estamos ante una "encarnación tecnológica", ante una experiencia extrasensorial en la que la sensación corporal es sustituida por abstracciones solo legibles instrumentalmente, corriendo el riesgo de que la tecnociencia dicte lo que realmente está pasando en los "cuerpos gestantes". Se produce así la ambivalencia entre lo real y lo imaginario, entre lo sensible y lo instrumental, induciéndose una contradicción entre las propias sensaciones maternas y lo que dictan las máquinas: "Si yo te estoy diciendo 
que tengo un dolor intenso, que el monitor es una máquina y a lo mejor va más lento" ( $x x \lambda)$. Se trata de la confusión entre la biometría y el propio ser materno, laberinto que desde la perspectiva medicalizada tiende a resolverse en favor de la primera. Los fundamentos ideológicos de esta realidad habría que buscarlos, como afirma Ballesteros (2007) y Fernández (2007), en la asunción de una ideología "posthumanista" que rechaza las posibles deficiencias físicas y morales del ser humano, buscando su superación a través de la exaltación de la tecnología. Partiendo de estas premisas se pretende "utilizar la tecnología para potenciar el cuerpo humano, curándolo, de una vez por todas de sus debilidades constitutivas" (Saraceni 2007: 145).

Las madres, conectadas a unos artefactos cuya exégesis tecnológica desconocen, tienden a delegar el propio poder a los especialistas, revelándose la tecnología como una de las realidades que mejor refleja el dominio de estos (Jordan 1993): "Pienso que estás más controlada, no sé, es que tampoco lo entiendes, si te ponen otra cosa, no sé, piensas que todo es bueno porque te van controlando" (xxK). Se produce así, como apunta Imaz (2002), la paradoja de que el portavoz del bebé sea en adelante la realidad dictada por las máquinas, quedando a un lado las sensaciones maternas. De esta manera, las madres asumen e interiorizan que los expertos dicten el estado de la "cuestión" por la seguridad que les aporta el control biotecnológico de sus partos: "Los monitores pues [...] hay que estar controlándoles a ellos porque en cualquier momento se pueden dar la vuelta y liarse con el cordón y si no lo tienes puesto pues no sabes lo que están haciendo" (xxع). En este sentido, parece que la tecnología, como afirman Ginsburg y Rapp (1995), se convierte, en cierta medida, en un instrumento de control de los profesionales, e incluso en un instrumento social, político y económico.

Se trata de toda una cosmovisión en la que la integridad de las madres y sus bebés, ha puesto todas sus expectativas en el control de las máquinas. Por ello, su uso es aceptado unánimemente al aportar seguridad a las madres: "Sabes que tienes que tenerlo porque eso es una seguridad para ti y para el bebé, entonces hay que ser conscientes de que necesitas un aparataje que controle el tema" (xxß). Estamos ante una representación de la dimensión corporal materna como vulnerable, por lo que las madres ya no quieren identificarse con ella (Viola 2007). Como apunta Montes (2009), los discursos del riesgo, y el miedo a la incertidumbre, justifican el uso de las técnicas, y las mujeres los han asumido como miembros de una matriz social compartida: "Sí, todo eso, todo eso es necesario [...] si no tienes ahí el aparato, el aparato puesto por decirlo así puede pasar cualquier cosa y no te enteras, teniendo así lo tienes controlado" (xxع). Ello lleva a demandar el uso de la tecnología, "por lo que puede implicar tener una criatura con discapacidad y por la tranquilidad que aportan" (Press et al. 1996: 52-53): "Pero a mí no me importaba, al contrario, al escuchar el monitor yo sabía que el niño estaba bien y estaba yo también controlándome" ( $x x \omega)$. Estamos ante una necesidad cultural, ante la aceptación total de la tecnología, ya que, a pesar de los inconvenientes y limitaciones de la misma, otorga a las madres una sensación de seguridad e infalibilidad (Goberna, Palacios y Banús 2008). Y es que, tal como afirma Saraceni (2007), la tecnología en sí misma no es algo perverso sino que se trata de algo lícito que sirve al ser humano para vencer sus propias deficiencias evolutivas. Hay que distinguir por tanto, en línea con lo apuntado por Habermans (2002), entre los usos humanistas e inhumanistas de la tecnología.

\section{"Abuso institucional"}

El término "abuso institucional" hace referencia a los diferentes agravios y conductas irrespetuosas que sufren las madres, respecto a su dignidad, durante el itinerario asistencial de sus partos (Blázquez 2009; Montes 2007; Esteban 2001). Este se produce en la medida en que las parturientas son impulsadas "para que interioricen su rol de pacientes, reforzando su socialización como subordinadas en el sometimiento y aceptación de los mandatos y deseos de los demás como propios" (Blázquez 2009: 291). Se trata de una realidad que va más allá de "simples desviaciones o imperfecciones del sistema "sanitario", ya que es una cuestión estructural al propio sistema que hay que contextualizar y repasar en su totalidad" (Esteban 2001: 35). Las causas hay que buscarlas en la propia dinámica asistencial de esta "etno-obstetricia" que disuelve, en cierta medida, el protagonismo de las madres al incorporarse a las rutinas del orden clínico preestablecido. Estamos ante una organización laboral impregnada de filosofías tayloristas ${ }^{9}$ y productivistas, que organiza el itinerario asistencial como si de una cadena de producción se tratara. Ello hace que, a veces, nuestra tipología de madres se sienta como una pieza más del engranaje asistencial: "Como que te identifican como uno más, otra persona más y un número más" (xxT); "has pasado ahora mismo y no me saludas [...] por lo menos gente que diga buenos días, hola" (xxw). Como comentábamos, estamos ante una relación asistencial asimétrica que da prelación a las necesidades clínicas, perdiendo las madres cierto poder de decisión: "Decisiones tú no puedes tomar ninguna, las decisiones las toman ellos, tú, yo es

9 El término taylorismo es un derivado del nombre del ingeniero mecánico y economista estadounidense Frederick Winslow Taylor (1856-1915). Hace referencia a la organización del trabajo caracterizada por la división, y asignación de tiempos, a las distintas tareas del proceso de producción. Se trata de una organización laboral íntimamente unida con la producción en cadena. 
que estás en un sitio que tienes que hacer lo que ellos hacen, lo que ellos te hagan" (xxy). Cuando se analizan los testimonios de las madres, subyace una línea discursiva que relaciona el "abuso institucional" con el hecho de ser diana de ciertas praxis esporádicas como las amenazas o agresiones verbales, la tendencia a la cosificación materna, la falta de respeto a la intimidad personal, las experiencias dolorosas, el ambiente desagradable, los abusos de autoridad y el aislamiento de los seres queridos. Hagamos, pues, un repaso de estas experiencias de "abuso institucional".

Una de las vivencias relatadas por las madres es el sometimiento a coacciones verbales por parte del personal. Se trata de conductas cuyo fin es conducir y convocar a las madres al cumplimiento de las normas clínicas preestablecidas, aunque de ello se derive cierta ofensa a su dignidad: "A mí hubo un momento personalmente en el que yo creo que no se me respetó mi dignidad, porque hubo un momento en que una de las asistentes que había allí me gritó, me habló de muy malas maneras, estando yo fatal como ya estaba de últimas, y creo que no se me respetó como se me tenía que haber respetado" (xxס). Estos abusos institucionales transforman, a veces, a las madres en objetos de intervención sobre los que se aplica el protocolo asistencial: "Es que ni mirándote, es que yo pienso que ni me miró a la cara como aquel que dice, o sea no, se echó encima y no le di porque no podía, no tenía fuerzas, ni podía ni decirle nada porque no tenía fuerzas, pero nada ni explicarte, ni decir que oye que te voy a empujar, tranquila, duele mucho pero bueno, nada absolutamente nada, es que nada ni buenas noches, ni buenos días, ni nada" (xxK). Ante estas praxis esporádicas las madres se sienten como objetos dejados por alguien, como entes orgánicos expuestos al personal para facilitar la rápida intervención. Los testimonios hablan, en ocasiones, de una dinámica fabril que no tiene tiempo para los aspectos emocionales, lo importante es la rutina del trabajo, soslayándose, a veces, aspectos tan importantes como la intimidad (Castro y Muñoz 1999): "Yo sinceramente [...] en la sala de dilatación, a veces, corrían la cortina y a veces no, las puertas abiertas, hombre una está en la situación que está [...] pero la intimidad es la intimidad y luego a la hora de dar a luz me encontré que tenía las dos mujeres [...] que me tocaron asistir al parto [...] y detrás tenía a siete mirando [...] hablando de sus cosas, y yo pues eso pues reventándome de dolor [...] entonces siete allí delante que las conté por gusto, o sea y hablando de sus cosas allí pues no, parecía que estaba en la prensa, ¿sabes? igual, me sentí en una situación muy incómoda" (xXK). Contexto en el que algunas madres relatan una especie de catarsis corporal, en la que las experiencias dolorosas son interpretadas como parte "normal" del ritual. Como apunta Montes (2007), el trance hacia la maternidad está impregnado de nociones expiatorias, por lo que la conversión total necesita de cierta purificación materna. Las madres perciben que los profesionales no admiten suficientemente el dolor infligido, etiquetando, a veces, a las pacientes como "quejicas": "Una exploración, que de verdad no se me olvida, porque, no sé, notaba mucho dolor [...] e incluso le dije que por favor parara" (xxт); "yo decía se creerán que soy quejica, de decir: ah, me duele, me duele, yo de verdad que no lo soy" ( $x x \lambda)$. Algunos testimonios revelan una atmósfera clínica impregnada de ruido, los técnicos van y vienen, ejecutando sus tareas sobre las madres, a la vez que mantienen conversaciones intrascendentes. Situación que es percibida por las madres como una falta de sensibilidad hacia ellas: "Ha habido cosas que no proceden, pues no, en las conversaciones y cosas que no, que uno tiene que estar, uno está allí dilatando y estás pariendo y estás así y las demás están hablando que si el niño tal y no sé cuánto [...] tenían que ser un poquito más humanos y mirar más por el paciente, sobre todo en esa profesión" (xxK). Ciertas mujeres se sienten expuestas al autoritarismo de los especialistas, "a las profundas idiosincrasias de las diferentes personalidades, valores e ideologías de los profesionales" (Montes 2007: 306), produciéndose un sentimiento de indefensión ante la asimetría de la interacción asistencial: "Dijo yo soy la que mando y ya está, que hay formas y formas, y se notó que en las formas no lo decía bien [...] claro lo que más me fastidió fue eso, porque yo digo a ti te dan una razón lógica [...] pero simplemente el que te digan [...] porque sí"

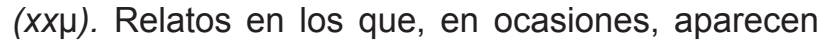
experiencias de soledad, al ser las madres incluidas en una especie de cadena asistencial productivista que las aísla de la sociedad y de sus propios seres queridos: "Luego no poder ver a mi marido aunque sea por la puerta, aunque sea verlo, que parece que te alivia, y tener al resto de, de personal ahí hablando de sus cosas, pues mal [...] o sea, a él no lo dejan entrar porque tienen que sacarme al nene con ventosa y tengo a siete, [...] y tres o cuatro detrás hablando de sus [...] historias [...] es que por favor, no procede, no procede, no" (xxK). Da la impresión de que la dinámica asistencial mecanizada no entiende, en demasiadas ocasiones, de afectividades, lo importante es el protocolo, el cumplimiento de las rutinas, la dimensión biológica del proceso. Praxis que suscitan en las madres ciertos sentimientos de humillación, inferioridad e impotencia: "No tienes, no sé, no te atreves a preguntar nada porque ves que te va a soltar un estufido" (xx $)$. Parece que se trata, como apuntan autores como Blázquez (2009), de una dinámica del trabajo íntimamente ligada a la filosofía productivista, desde la que se da prelación a la intervención técnica sobre el componente emocional. Lo importante, pues, es la obtención de un producto sano, el bebé, en el menor tiempo y con las menores interferencias posibles. En este sentido, y salvando las distancias, los paritorios se configuran 
como una cadena industrial donde la accesibilidad del producto sobre el que se interviene, el ruido y las rutinas de trabajo se convierten en lo prioritario, tendiendo a convocar a las madres para que se ajusten al proceso ritual, y a los acompañantes para que no interfieran en las rutinas fabriles preestablecidas.

Como apunta Habermans (2002), estamos ante un cierto olvido de las necesidades emocionales de las madres que restringe la simetría de la responsabilidad existente entre personas libres e iguales. Realidad que, a veces, forma parte estructural de la asistencia, y que ha sido asumida por la mayoría de gestantes durante el itinerario asistencial. Ello, junto con los aspectos positivos de la tecnología, no hay que olvidar que minimiza riesgos y aporta seguridad a las madres, hace que estas rara vez tengan conciencia de ser objeto de abusos, y aunque "la mayoría [...] guardan recuerdos de sufrimiento [...] no tiene conciencia de los posibles abusos recibidos" (Gutman 2006: 295): "La asistencia bien, en la mayoría, bueno, la mayoría muy bien, la verdad [...] sí la verdad es que sí, bastante bien, bastante bien" (xхK) .Se produce, así, en general, una aceptación e identificación de las definiciones y características del proceso, que además tiene lugar en el hospital, "templo de la eficiencia y la eficacia" (Comelles 1996: 330) de los profesionales. Aceptación con la que las madres expresan su satisfacción al haber salido de este "rito de paso" intervencionista con el resultado esperado, ellas y sus criaturas sin más perjuicios que los ya previstos con antelación. Como diría Beck (1999), se perpetúan así ciertas características del modernismo como la confianza absoluta en los sistemas de expertos derivados de las ciencias empíricas. La consecuencia final es la invisibilización del "abuso institucional", a pesar de ser este un tema conocido ${ }^{10}$ y reiterado en el propio relato de las madres. Se trata de una especie de "amnesia retrógrada", por la que la aceptación de cierto grado de abuso y autoritarismo como lo "normal", junto con los beneficios de la tecnología y el consenso social que justifica cualquier actuación clínica como necesaria en pro de la disminución del riesgo, dificulta su visualización.

\section{Conclusiones}

Los relatos de la tipología de madres seleccionadas para este estudio reflejan toda una serie de "mundologías" y conflictos asistenciales fruto de la tensión surgida entre sus vivencias y las rutinas medicalizadoras propias de esta "etnoobstetricia". Conflictos entre los que destacan experiencias como la interacción asistencial asimétrica, la subordinación tecnológica, o el "abuso institucional". Los resultados

10 El "abuso institucional" o "violencia institucional" durante los partos ha sido denunciado, desde la perspectiva de género, por antropólogas como María Luz Esteban (2001b), María Jesús Montes (2007) o María Isabel Blázquez (2009). muestran un ritual que parece impregnado de nociones de riesgo y sumisión, propiciando en la mayoría de ocasiones el sometimiento y la aceptación de las madres del estudio a las rutinas clínicas, aunque ello suponga la adopción de un papel pasivo desde el que cobra especial protagonismo la tecnología y el control profesional. Se dejan ver aquí los efectos del productivismo, en el sentido de que, a veces, se da mayor prelación a las rutinas clínicas que a las propias necesidades maternas. Control y mecanización asistencial que aporta, no obstante, una mayor seguridad a las mujeres investigadas, sirviendo de basamento para la perpetuación de un modelo asistencial que, a veces, es resignificado desde su propia óptica, al haber asumido como positivo el férreo control al que son sometidas durante el itinerario asistencial. Parece que estamos ante unas perspectivas asistenciales impregnadas por la "biopolítica", mecanismo estatal para garantizar la reproducción de ciudadanos sanos y productivos.

En los relatos subyace, también, cierta desconfianza hacía sus propios cuerpos, que, en ocasiones, perciben como caprichosos e imprevisibles, produciéndose, así, cierto grado de enculturación del proceso en clave patológica. Desde esta óptica, parece que se produce un alto grado de aceptación de la tendencia controladora e intervencionista típica de este paradigma asistencial. Consentimiento que en su mayoría otorgan las madres del estudio en favor de una supresión de los riesgos de que se han hecho coparticipes, a pesar de que ello suponga cierta pérdida de control sobre su propio proceso de parto. Se trata de un proceso altamente tecnológico que ha puesto sus expectativas en ciertas representaciones "posthumanistas" que, en aras de una mayor seguridad, quisiera traducir las experiencias humanas en simples datos biométricos.

De los testimonios de las madres de esta investigación se deduce que el miedo al peligro ha inmovilizado, en parte, sus recursos y saberes, moldeando sus voluntades, por lo que se ponen en manos de los "expertos" para someterse a un ritual iniciático que es justificado y vivido como lo deseable y "normal". En este "habitus"11 las experiencias desagradables, el "abuso institucional", e incluso, a veces, la capitulación de ciertas dignidades, es opacado y asumido en favor de la seguridad que aporta la tecnología, surgiendo en los alegatos maternos esa tensión apuntada entre sus necesidades emocionales y la tendencia medicalizadora y biologicista, típica de este patrón asistencial. Pese a sus inconvenientes, se trata de una "cultura del nacimiento" que sigue en plena vigencia en los hábitats clínico-hospitalarios de las socie-

11 El "habitus" es uno de los conceptos centrales de la teoría sociológica de Pierre Bourdieu. Por tal podemos entender 'esquemas de obrar, pensar y sentir asociados a la posición social, que hacen que personas de un entorno social homogéneo tiendan a compartir concepciones y estilos de vida parecidos'. 
dades postmodernas contemporáneas, seguramente como testimonio de la persistencia de ciertos "metarrelatos modernistas" que sostienen su confianza absoluta en los sistemas profesionales derivados de las ciencias positivas. En este sentido, parece que en un futuro habrá que introducir elementos de reflexión que apuesten por una mayor humanización de los partos institucionalizados, intentando así aunar las necesidades maternas con los intereses institucionales y burocráticos que apuestan por el intervencionismo biomédico como medio de control de riesgos. Por tanto, es preciso vertebrar nuevos marcos conceptuales desde los que interpretar el nacimiento, generando posibilidades donde no las hay, y articulando nuevas inquietudes y representaciones que hagan a los sistemas sanitarios más permeables a las necesidades y conflictos de las madres, sin abandonar por ello los beneficios y la seguridad del control biomédico.

\section{ReferenCIAS BibLIOGRÁfiCAS}

Amato, A. C. 2007. "El cuerpo glorioso, entre representación y experimentación". Pp. 63-80 en Biotecnología y Posthumanismo, coordinado por J. Ballesteros y E. Fernández. Navarra: Aranzadi.

Ballesteros, J. 2007. "Biotecnología, Biolítica y Posthumanismo”. Pp. 21-46 en Biotecnología y Posthumanismo, coordinado por J. Ballesteros y E. Fernández. Navarra: Aranzadi.

Baroni, F., L. Vilela y F. Scorsolini. 2013. "Significados da gravidez e da maternidade: discursos de primíparas e multíparas". Psicología: teoría e prática 15:19-34.

Beck, U. 1999. Word Risk Society. Stafford: Polity Press.

Bertaux, D. e I. Bertaux. 1993. "Historias de vida del oficio de panadero". Pp. 231-250 en La historia oral: Métodos y experiencias, compilado por J. M Marinas y C. Santamaría. Madrid: Debate.

Bezerra, M. G. y M. V. Cardoso. 2005. "Factores que interfieren en el comportamiento de las parturientas: enfoque en la etnoenfermería". Rev. Bras. Enferm 58:698-702. http://dx.doi.org/10.1590/ S0034-71672005000600013

Blázquez, M. L. 2009. “Ideologías y prácticas de género en la atención sanitaria del embarazo, parto y puerperio: el caso del área 12 de la Comunidad de Madrid". Tesis Doctoral, Universidad d Rovira i Virgili, Tarragona.

Bodoque, Y. 1996. "Discursos y prácticas sobre sexualidad y reproducción". Tesis doctoral, Universidad Rovira i Virgili, Tarragona.

Borrell, C. 2004. "Desigualdades en salud según la clase social en las mujeres. ¿Cómo influye el tipo de medida de la clase social?". Gaceta Sanitaria 18:75-82. http://dx.doi.org/10.1157/13061997

Bourdieu, P. 1994. Por una sociología reflexiva. Barcelona: Herder.

Bravo, P., C. Uribe y C. Contreras. 2008. "El cuidado percibido durante el proceso de parto: una mirada desde las madres". Rev. Chil. Obstet, ginecol 73:179-184. http://dx.doi.org/10.4067/ S0717-75262008000300007
Campbell, R. and A. Macfarlane. 1987. Where to be born?. Oxford: Nacional Perinatology Epidemiology Unit.

Castro, A. y A. Mu-oz. 1999. "Experiencia de primíparas en su trabajo de parto: Maternidad del Hospital Dr. Sotero del Río". Tesis para optar al título de enfermera matrona, Pontificia Universidad Católica de Chile, Santiago.

Comelles, J. M. ${ }^{a}$. 1996. "Fe, carisma y milagros. El poder de curar y la sacralización de la práctica médica contemporánea". Pp. 301-336 en Creer y curar: la medicina popular, editado por José Antonio González Alcantud y Salvador Rodríguez Becerra. Granada: Biblioteca de Etnología Diputación Provincial de Granada.

Cunha, C. y C. L. Ojeda. 2006. "Enfermería y apoyo emocional: la perspectiva de la parturienta". Revista de Psicología de la Universidad de Chile 1:55-71.

Davis-Floyd, R. 2001. The technocratic, humanistic, and holistic paradigms of child-birth. International Journal of Gynecology \& Obstetrics 75:5-23. http://dx.doi.org/10.1016/ S0020-7292(01)00510-0

Duden, B. 1996. "El concepto de vida: un ídolo moderno y una amenaza para las mujeres embarazadas". Douda 11:79-96.

Esteban, M. L. 1992. "Actitudes y percepciones de las mujeres respecto a su salud reproductiva y sexual. Necesidades de salud percibidas por las mujeres y respuesta del sistema sanitario." Tesis doctoral, Universidad de Barcelona, Barcelona.

Esteban, M. L. 2001. "El género como categoría analítica. Revisiones y aplicaciones a la salud". Pp. 25-51 en Perspectivas de género en salud, compilado por Miqueo et al. Fundamentos científicos y socioprofesionales de diferencias sexuales no previstas. Madrid: Minerva.

Federación de Asociaciones de Matronas de España, FAME. 2008. Documento de iniciativa a favor del parto normal. Madrid: Autor.

Fernández, E. 2007. "Crítica filosófica del posthumanismo: Gabriel Marcel". Pp. 81-108 en Biotecnología y Posthumanismo, coordinado por J. Ballesteros y E. Fernández. Navarra: Aranzadi.

Ferrer, U. 2007. "Posthumanismo y dignidad de la especie humana". Pp. 153-170 en Biotecnología y Posthumanismo, coordinado por J. Ballesteros y E. Fernández. Navarra: Aranzadi.

Foucault, M. 1990. La vida de los hombres infames. Madrid: La Piqueta.

Ginsburg, F. y R. Rapp. 1995. Conceiving the New World Order: The Global Politics of Reproduction. Berkeley: University of California Press.

Goberna, J, A. Palacio y M. R. Banús. 2008. "Tecnología y humanización en la asistencia al nacimiento. La percepción de las mujeres". Matronas Prof. 9:5-10.

Grele, R. J. 1991. "La historia y sus lenguajes en la entrevista oral, quién contesta a las preguntas de quién y por qué". Historia y Fuente oral 5:106127.

Gutman, L. 2006. La maternidad y el encuentro con la propia sombra. Barcelona: Editorial del Nuevo Extremo. RBA. Integral. 
Habermans, J. 2002. El futuro de la naturaleza humana. ¿Hacia una eugenesis liberal?. Barcelona: Paidós.

Hernández Garre, J. M. 2011. "El parto hospitalario e intervencionista. Una construcción social de la maternidad". Tesis Doctoral, Universidad de Murcia, Murcia.

Hodnett, E. D, S. Gates, G. J. Hofmeyr, C. Sakala and J. Weston. 2011. "Continuous support for women during childbirth". Cochrane Database of Systematic Review, Issue 3. Art. No.: CD003766. http://dx.doi.org/10.1002/14651858. CD003766.pub3

Ibáñez, J. 1979. "Interpretación y análisis del discurso". Pp. 333-351 en Más allá de la sociología. El grupo de discusión, técnica y crítica, editado por Ibáñez, J. Madrid: Siglo XXI.

Imaz, E. 2002. "Tres lecturas del cuerpo materno: antojos, anemias y ecografías". Paper presentado en el IX Congreso de Antropología de la Federación de Asociaciones de Antropología del Estado Español, 4-7 de septiembre, Barcelona.

Imaz, E. 2008. "Mujeres gestantes, madres en gestación. Representaciones, modelos y experiencias en el tránsito a la maternidad de las mujeres vascas contemporáneas". Tesis doctoral, País Vasco, Universidad del País Vasco.

Joaquim, T. 1983. Dar à luz. Ensaio sobre as práticas e crenças de gravidez, parto e pós-parto em Portugal. Lisboa: Publicaçoes Dom Quixote.

Jociles, M. ${ }^{a}$ I. 2005. "El análisis del discurso: de cómo utilizar desde la antropología social la propuesta analítica de Jesús Ibáñez". Revista de Antropología 7:1-25.

Jordan, B. 1993. Birth in four cultures. A Crosscultural Investigation of Childbirth in Yucatan, Holland, Sweden and the United States. Montreal: Eden Press Women's Publications.

Juan, M. 1991. "Crear el nacimiento: la medicalización de los conflictos en la reproducción". Revista Española de Investigaciones Sociológicas 53:29-51. http://dx.doi.org/10.2307/40183575

Luque, M. A. y M. I. Oliver. 2005. "Diferencias culturales en la percepción y vivencia del parto: el caso de las mujeres inmigradas". Año XIV 48-49:9-13.

Maroto, G; E. Casta-o, M. M. García, N. Hidalgo e I. Mateo. 2009. "Paternidad y servicios de salud. Estudio cualitativo de las experiencias y expectativas de los hombres hacia la atención sanitaria del embarazo, parto y posparto de sus parejas". Rev. Esp. Salud Publica 83:267-278. http://dx.doi.org/10.1590/S113557272009000200010

Mauss, M. 1966. Sociologie et anthropologie. París: PUF.

Martin, E. 1987. The Woman in the Body. Boston: Beacon Press.

McClain, C. 1975. "Ethno-obstetrics in Ajijic". Anthropological Quarterly 48:38-56. http:// dx.doi.org/10.2307/3316856

Menéndez, E. L. 2000. "Factores culturales. De las definiciones a los usos específicos". Pp. 163188 en Medicina y Cultura. Estudios entre la Antropología y la Medicina, editado por Perdiguero y Comelles. Barcelona: Bellaterra.

Menéndez, E. L. 2002. La parte negada de la cultura. Barcelona: Ediciones Bellaterra.
Menéndez, E. L. y R. di Pardo. 1996. De algunos alcoholismos y algunos saberes. Atención primaria y proceso de alcoholización. México: Casa Chata.

Merighi, M. A, G. M. Carvalho y V. P. Suletroni. 2007. "El proceso del parto y nacimiento: visión de las mujeres con convenio de salud en la perspectiva de la fenomenología social". Acta Paul. Enferm 20:434-440.

(De) Miguel, J. 1979. El mito de la inmaculada concepción. Barcelona: Anagrama.

Ministerio de Sanidad y Política Social, MSPS. 2010a. Guía de Práctica Clínica sobre la atención al parto normal. Madrid: Autor.

Ministerio de Sanidad y Política Social, MSPS. 2010b. Estrategia Nacional de Salud Sexual y Reproductiva. Madrid: Autor.

Montes, M.a J. 2007. "Las culturas del nacimiento. Representaciones y prácticas de las mujeres gestantes, comadronas y médicos". Tesis doctoral, Univesitat Rovira i Virgili, Tarragona.

Narotzky, S. 1995. Mujer, mujeres, género. Una aproximación crítica al estudio de las mujeres en las ciencias sociales. Volumen 14, Monografías. Madrid: Editorial CSIC.

Oakley, A. 1984. The captured Womb: a history of the Medical Care of Pregnant Women. Oxford: Basil Bernstein.

Odent, M. 1990. El bebé es un mamífero. Madrid: Mandala.

Odent, M. 2011. El nacimiento en la era de plástico. Tenerife: Ob Stare.

Olsen, O. 1997. "Meta-analysis of the safety of Home Birth". Birth; Issues in perinatal care 24:4-13. http:// dx.doi.org/10.1111/j.1523-536X.1997.tb00330.x

Organización Mundial de la Salud, OMS. 1985. Declaración de Fortaleza. Tecnología apropiada para el parto. Ginebra: Autor.

Organización Mundial de la Salud, OMS. 1996. Cuidados en el parto normal: una guía práctica. Ginebra: Autor.

Ortiz, T. 2006. Medicina, historia y género, 130 años de investigación feminista. Oviedo: KRK.

Osorio, R. M. 2001. Entender y atender la enfermedad. Los saberes maternos frente a los padecimientos infantiles. México: CIESAS, INAH, INI.

Pizzini, F. 1981. "Il parto in ospedale: tragitto della donna e rituali dell'istituciones". Pp. 129-147, en Sulla scena del parto: luoghi, figure, pratiche, coordinado por F. Pizzini. Milano: Franco Angeli Editore.

Press, N., C. Browner, D. Tran, C. Morton and B. Le Master. 1996. "Provisional normality and perfect babies: pregnant women's attitudes towards disability in the context of the prenatal testing". Pp. 46-65 in Reproducing Reproduction, edited by S. Franklin y H. Ragone. Philadelphia: University of Pennsylvania Press.

Romaní, O. 1992. "Marginación y drogodependencias. Reflexiones en torno a un caso de investigación". Pp. 259-281 en Marginación e inserción. Los nuevos retos de las políticas sociales, compilado por S. Franklin y H. Ragone. Madrid: Eudymión.

Saraceni, G. 2007. "El cuerpo del delito. Reflexiones jurídico-filosóficas sobre el posthumanismo". Pp. 139-152 en Biotecnología y Posthumanismo, coordinado por J. Ballesteros y E. Fernández. Navarra: Aranzadi. 
Schwarz, P. 2008. "Las lesbianas frente al dilema de la maternidad". Pp. 193-214 en Todo sexo es político, coordinado por M. Pecheny, C. Figari y D. Jones. Buenos Aires: Del Zorzal.

Sociedad Española de Ginecología y Obstetricia, SEGO. 2008a. Recomendaciones sobre la asistencia al parto normal. Madrid: Autor.

Sociedad Española de Ginecología y Obstetricia, SEGO. 2008b. Riesgos clínicos-laborales y embarazo. Madrid: Autor.

Stoller, N. 1974. Forced labor: maternity care in the United States. Oxford: Pergamon Studies in Critical Sociology.

Suárez, S. y M. Puertas. 2008. "Vivencias de la mujer embarazada con hipertensión arterial". Biblioteca Las Casas 4:1-6.

Taussing, M. 1995. Un gigante en convulsiones. El mundo humano como un sistema nervioso en emergencia permanente. Barcelona: Gedisa.

Taylor, S. J. y R. Bogdan. 1987. Introducción a los métodos cualitativos de investigación: la búsqueda de significados. Barcelona: Paidós.
Tew, M. 1985. "Place of birth and perinatal mortality". J R Coll Gen Pract 35: 390-394.

Viola, F. 2007. "La defensa de la persona humana en la era tecnológica". Pp. 47-62 en Biotecnología y Post-humanismo, coordinado por J. Ballesteros y E. Fernández. Navarra: Aranzadi.

JOSÉ MANUEL HERNÁNDEZ GARRE es Doctor en Antropología social (Facultad de Filosofía de la Universidad de Murcia). Profesor de la Universidad Católica San Antonio. Grupo investigación: Pensamiento en el contexto social. Línea de investigación: Antropología de la salud.

PALOMA ECHEVARRÍA PÉREZ es Doctora en Antropología social (Universidad Católica de Murcia). Decana de la Facultad de Enfermería de la Universidad Católica San Antonio. Grupo investigación: Pensamiento en el contexto social. Línea de investigación: Antropología de la salud. 